\title{
Estatura de adolescentes matriculados em escolas da rede pública no estado do Paraná, Brasil
}

\author{
Height of adolescents enrolled in public \\ schools in Paraná state, Brazil
}

Suely Teresinha Schmidt Passos de AMORIM ${ }^{1}$

Alline Gouvea Martins RODRIGUES²

Márcia Cristina STOLARSKI ${ }^{3}$

\section{RE S U M O}

\section{Objetivo}

Avaliar o crescimento de adolescentes matriculados na rede pública no Paraná, comparando com os dados da Pesquisa Nacional sobre Saúde e Nutrição Região Sul, e identificar a ocorrência de déficit de estatura pela comparação com valores de referência.

\section{Métodos}

Avaliaram-se 13216 escolares da rede pública no Paraná entre 10 a 18 anos, sendo 6323 do sexo masculino e 6893 do sexo feminino. A definição da amostra foi por conveniência. A análise da distribuição estatura para idade foi realizada com curvas de probabilidade normal e estatisticamente com o teste Kolmogorov-Smirnov. Para a comparação com os dados da Pesquisa Nacional sobre Saúde e Nutrição Região Sul e com os valores de referência do Center for Disease Control and Prevention, 2000, foi desenvolvida uma curva de crescimento.

\section{Resultados}

A estatura para idade entre os escolares obedece a uma distribuição normal em todas as faixas etárias e em ambos os sexos. A prevalência de baixa estatura é muito próxima dos valores esperados para uma população normal. A mediana da estatura é maior em relação à Pesquisa Nacional sobre Saúde e Nutrição Região Sul, em todas as idades e em ambos os sexos. Déficits de estatura de 0,05 a 2,39 cm foram constatados nas faixas etárias dos 12 aos 18 anos.

\section{Conclusão}

As diferenças positivas na estatura dos escolares em relação aos dados da Pesquisa Nacional sobre Saúde e Nutrição Região Sul, e os pequenos déficits em relação aos valores de referência, sugerem que melhorias

\footnotetext{
1 Universidade Federal do Paraná, Departamento de Nutrição. Campus III, Av. Prefeito Lothário Meissner, Jardim Botânico, 632, 82010-170, Curitiba, PR, Brasil. Correspondência para/Correspondence to: S.T.S.P. AMORIM. E-mail: <samorim@ufpr.br>.

2 Universidade Federal do Paraná, Curso de Especialização em Nutrição Clínica, Departamento de Nutrição. Curitiba, PR, Brasil.

3 Secretaria de Estado da Educação do Paraná. Curitiba, PR, Brasil.
} 
nas condições sociais e econômicas do Paraná possam ter contribuído com esses resultados. Entretanto, diferenças regionais deverão ser consideradas para o melhor planejamento de políticas públicas.

Termos de indexação: Adolescentes. Antropometria. Avaliação nutricional. Crescimento. Estatura.

\section{A B S T R A C T}

\section{Objective}

The objective of this study is to assess the growth of adolescents enrolled in the public school system of the State of Paraná and compare the data with that of the National Health and Nutrition Survey South Region and to identify the occurrence of stunting by comparison to reference values.

\section{Methods}

A total of 13,216 students were selected by convenience sampling. The sample consisted of 6,323 boys and 6,893 girls, aged 10 to 18 years. The height-for-age distribution was analyzed using normal plots and the Kolmogorov-Smirnov test. A growth curve was constructed for the students in Paraná in order to compare it with the values from the National Health and Nutrition Survey South Region and Center for Disease Control and Prevention, 2000.

\section{Results}

The height-for-age index of the students followed a normal distribution for all ages and both genders. The prevalence of stunting is very close to that expected for a normal population. The height median is higher than that of the National Health and Nutrition Survey South Region, for all ages and both genders. A stunting of 0.05 to $2.39 \mathrm{~cm}$ was found among the 12- to 18-year-olds.

\section{Conclusion}

The positive differences between the studied population and the data from the National Health and Nutrition Survey South Region, along with the small deficits when compared with the Center for Disease Control and Prevention reference values suggest that improvements in the social and economic conditions of Paraná may have contributed to these results. Regional differences must be considered for better planning of public policies.

Indexing terms: Adolescents. Nutrition assessment. Anthropometry. Body height. Growth.

\section{N T R O D U Ç Ã O}

Com algumas exceções, estima-se que as populações têm potenciais genéticos para atingirem um intervalo de crescimento similar. Alterações na estatura final, nas taxas de crescimento e de desenvolvimento físicos, podem ser atribuídas às diferenças ambientais, vistas não apenas em seu aspecto físico, mas às condições sociais, econômicas, de saúde e nutrição ${ }^{1,2}$.

A situação socioeconômica pode propiciar uma melhor qualidade de vida, possibilitar o bem-estar nutricional, modificar os padrões de exposição e risco das doenças e permitir o desenvolvimento do potencial genético de crescimento linear dos indivíduos ${ }^{1,3}$.
Os resultados de dois grandes estudos nacionais o Estudo Nacional da Despesa Familiar (ENDEF), realizado em 1974, e a Pesquisa Nacional sobre Saúde e Nutrição (PNSN), em 1989, mostram que houve uma tendência secular em estatura positiva, em todas as regiões do Brasil| .

O fenômeno de Tendência Secular em Estatura (TSE) é definido como uma alteração no perfil de crescimento da população, resultante de modificações, no decorrer do tempo, na idade em que determinadas estaturas são atingidas por crianças ou adolescentes, ou então, de alterações na altura final alcançada por uma população adulta $^{5}$.

Estudos sobre mudanças seculares na estatura podem fornecer dados acerca do estado 
nutricional na infância e auxiliar na interpretação de padrões do crescimento. Contribuem também para caracterizar o desenvolvimento socioeconômico da população, visto que o fenótipo humano é extremamente plástico e o crescimento físico é particularmente sensível à qualidade de seus ambientes sociais e econômicos. Sabe-se que crianças com atraso do crescimento por condições socioeconômicas inadequadas, podem se transformar em adultos insalubres, com capacidades intelectuais prejudicadas e limitado potencial de desenvolvimento ${ }^{1,3}$.

Portanto, para que a saúde seja mantida e as crianças e adolescentes consigam atingir um desenvolvimento futuro pleno nos termos da saúde geral e de produtividade, é de extrema importância que o crescimento físico seja adequado'. Do mesmo modo, é importante que o crescimento seja monitorado ou, pelo menos, avaliado com determinada freqüência.

Em algumas situações não se dispõe de dados acerca da estatura de uma população que permitam a comparação com dados atuais e, conseqüentemente, a análise da tendência secular do crescimento. Entretanto, é possível efetuar comparações entre resultados de pesquisas locais com dados de estudos nacionais ou regionais e apontar possíveis diferenças na estatura final dos indivíduos, alvo dessas pesquisas.

O Estado do Paraná não dispõe de investigações que permitam análises acerca da tendência secular do crescimento de sua população. Por isso, este estudo tem por objetivo avaliar e comparar a estatura de escolares da rede pública estadual do Paraná com dados da Pesquisa Nacional sobre Saúde e Nutrição ${ }^{6}$ Região Sul, detectando possíveis diferenças, e definindo a ocorrência de déficit de estatura pela comparação com valores de referência do National Center for Health Statistics (NCHS) - Centers for Disease Control and Prevention (CDC) ${ }^{7}$, além de verificar possíveis alterações no crescimento resultantes de discrepâncias socioeconômicas entre os municípios do Estado.

\section{MÉ T O D O S}

Trata-se de um estudo populacional transversal ou de prevalência, com 13216 escolares beneficiários do Programa Estadual de Alimentação Escolar (PEAE), no Estado do Paraná. Este estudo constitui parte de um projeto sobre a avaliação do estado nutricional de escolares matriculados na rede pública estadual no Paraná, uma parceria entre o Departamento de Nutrição da Universidade Federal do Paraná (UFPR) e o Instituto de Desenvolvimento Educacional do Paraná (FUNDEPAR), órgão vinculado à Secretaria de Estado da Educação.

A definição da amostra do estudo foi estabelecida pelo FUNDEPAR, que considerou a abrangência e as peculiaridades da clientela do PEAE no Estado do Paraná. A partir de um procedimento não probabilístico por conveniência, foram definidos o tamanho da amostra e a seleção das unidades de pesquisa (municípios e escolas) em cada Núcleo Regional de Educação (NRE).

Os NRE são unidades administrativas descentralizadas da Secretaria de Estado da Educação do Paraná, compostos por municípios geograficamente próximos, com função de atendimento às redes escolares (públicas, privadas e conveniadas) sob sua jurisdição.

Os municípios de cada NRE podem apresentar características socioeconômicas e demográficas distintas. Assim, considerando o número, a localização e as características dos municípios e das escolas públicas, foram selecionados pelo FUNDEPAR, três municípios, de diferentes portes, em cada um dos 32 Núcleos Regionais de Educação.

O porte dos municípios foi definido de acordo com o número de escolas da rede pública estadual: aqueles que apresentavam de duas a quatro escolas foram definidos como de pequeno porte; de médio porte aqueles que tinham de 5 a 10 escolas e; grande porte aqueles que tinham acima de 11 escolas. O porte dos estabelecimentos de ensino também foi definido pelo FUNDEPAR, em função do número de alunos matriculados e 
apresenta grande variação no Estado. Todas as escolas dos municípios de pequeno porte selecionados que apresentavam entre duas a três escolas fizeram parte da amostra. Em relação aos municípios de médio e grande porte, foram selecionadas: a escola com menor, aquela com número intermediário e aquela com o maior número de alunos. Portanto, a composição da amostra de todo o estudo, contemplou 99 municípios, 324 escolas e 14422 adolescentes e adultos jovens, na faixa etária de 10 a 25 anos, correspondendo a $23,6 \%$ dos municípios do Estado do Paraná, $20,2 \%$ do total de escolas, $1,8 \%$ dos escolares atendidos pelo Programa Estadual de Alimentação Escolar e 1,2\% dos escolares matriculados em Escolas Estaduais do Paraná em 2004.

Para o presente estudo foram selecionados, a partir dos dados compilados, adolescentes de 10 a 18 anos, totalizando 13216 indivíduos, sendo 6323 do sexo masculino e 6893 do sexo feminino, matriculados em 324 escolas dos 99 municípios propostos.

Os dados antropométricos foram coletados em 2004, sob a responsabilidade do Fundepar, por nutricionistas e acadêmicos de nutrição, previamente treinados. Todos os escolares foram pesados e medidos, sem calçados e vestindo apenas uma camiseta e bermudas.

Os dados antropométricos foram compilados utilizando-se o programa Epi Info versão $6 b c^{8}$. Fez-se dupla digitação dos dados por digitadores distintos, sendo essa avaliada com o módulo Validate do programa. Discrepâncias nos dados foram re-examinadas e corrigidas. As análises estatísticas descritivas foram efetuadas utilizando-se do aplicativo Win Stat para o Excel. Para a comparação com valores de referência mais atuais (CDC 2000) do que o utilizado na versão 6 bc do programa, os valores antropométricos de estatura para a idade foram re-analisados no Excel, utilizando a fórmula estatística: $=\mathrm{SE}(\mathrm{Aa}<=\mathrm{Bb}$; "baixa estatura"; (SE(Aa>Bb; "eutrófico")), segundo as quais:
$\mathrm{A} a=$ coluna e linha da estatura coletada

$\mathrm{Bb}=$ coluna e linha do escore $-2 \mathrm{Z}$ da curva do CDC - 2000.

Utilizou-se o índice estatura para a idade para avaliar a distribuição da estatura por faixa etária e sexo. Os valores resultantes foram comparados aos valores de referência para mesma idade e sexo do $\mathrm{CDC}^{7}$. A medida estatística utilizada foi o escore-Z, tendo como ponto de corte inferior de normalidade valores maiores que $-2 Z$ da mediana do CDC, e como limite superior de normalidade valores menores ou iguais a $2 Z \mathrm{da}$ mediana do $\mathrm{CDC}^{7}$. Valores abaixo ou iguais a $-2 Z$ foram classificados como baixa estatura.

A análise da distribuição do índice estatura para a idade foi realizada graficamente com curvas de probabilidade normal e estatisticamente com o teste de Kolmogorov-Smirnov ${ }^{9}$.

Para a construção dos grupos etários utilizou-se a idade centrada. A idade de 11 anos, por exemplo, correspondeu ao intervalo de 126 meses (10 anos e meio) a 137,99 meses (11 anos e meio). Este procedimento foi realizado para as idades entre 10 e 17 anos. Para a idade de 18 anos foi considerado o intervalo de 210 meses (17 anos e meio) até 216 meses (18 anos completo).

Para a comparação com as medidas de adolescentes das mesmas faixas etárias da PNSN - Região Sul e dos valores de referência do $\mathrm{CDC}^{7}$ foi desenvolvida uma curva de crescimento dos escolares paranaenses, a partir da mediana e dos percentis 5 e 95, para cada grupo etário e sexo. Optou-se pela apresentação das medianas por não serem afetadas por valores muito abaixo ou acima dos gerais, e também porque os demais valores usados para comparação, como os resultados do $\mathrm{CDC}^{7}$ e da PNSN, tratam-se de medianas. Contudo, os resultados da comparação entre médias e medianas obtidas para a população em análise foram semelhantes, alterando-se em média $0,34 \mathrm{~cm}$.

Para determinar possíveis alterações no crescimento decorrentes de diferenças socioeco- 
nômicas no Estado utilizou-se o diagrama de dispersão de estatura versus Índice de Desenvolvimento Humano (IDH) Municipal - IDH-M (2000) agrupado no valor central; por exemplo, o IDH de 6,50 corresponde a valores de estatura de escolares em municípios com IDH maiores ou iguais a 6,25 e inferiores a 6,75.

O estudo foi aprovado pelo Comitê de Ética do Setor de Ciências da Saúde da Universidade Federal do Paraná.

\section{RES U LTA DOS}

A freqüência de baixa estatura entre os escolares no Paraná, identificada pelo índice estatura para a idade, é apresentada na Tabela 1. Pela análise dos dados verifica-se dispersão em relação ao esperado na população de referência, que seria de 2,3\% abaixo de -2Z (baixa estatura). Aos 11 anos, a distribuição mantém-se abaixo do esperado para baixa estatura, com $2,16 \%$. Aos 10 e entre 12 e 18 anos a prevalência de baixa estatura, é maior do que o esperado, chegando a $5,63 \%$ aos 17 anos e 5,03\% aos 18 anos. Para o total de escolares avaliados, tem-se que a prevalência de baixa estatura é muito próxima da referência $\mathrm{CDC}^{7}$, sendo apenas $1,38 \%$ maior.

Pela distribuição da baixa estatura, por idade e sexo, verifica-se que, o déficit de estatura é superior no sexo masculino, em relação ao feminino, nas idades de 10 e dos 14 aos 18 anos. Entre os meninos, as prevalências de baixa estatura foram superiores ao esperado em todas as idades, exceto aos 11 anos, sendo que nas idades de 16, 17 e 18 anos é mais que o dobro do considerado normal (2,3\%) para a população de referência ${ }^{7}$. A prevalência mais alta foi de 7,1\%, encontrada aos 17 anos, ou seja, mais que o triplo do considerado normal (2,3\%) para a população de referência ${ }^{7}$. Entre as meninas, prevalências superiores ao esperado foram encontradas a partir dos 11 anos.

De um modo geral, as prevalências de déficit de estatura foram baixas: para o total de escolares, por sexo, foram 1,3\% e 1,4\% superiores aos $2,3 \%$ esperados, respectivamente, para meninas e meninos.

Para identificar se as distribuições dos valores de escore- $Z$ de estatura para a idade são normais, utilizou-se o teste de Kolmogorov-Smirnov. O resultado do teste indicou distribuição normal para ambos os sexos $(p>0,20)$. A representação gráfica dessa distribuição é feita na Figura 1.

Esta mesma análise foi feita para cada faixa etária em ambos os sexos e em todos os casos, pelo teste de Kolmogorov-Smirnov, $(p>0,20)$, encontraram-se distribuições normais.

Tabela 1. Frequência de baixa estatura para a idade em escolares segundo sexo e faixa etária. Paraná, 2004.

\begin{tabular}{|c|c|c|c|c|c|c|c|c|c|}
\hline \multirow{2}{*}{$\begin{array}{l}\text { Idade } \\
\text { (anos) }\end{array}$} & \multicolumn{3}{|c|}{ Feminino } & \multicolumn{3}{|c|}{ Masculino } & \multicolumn{3}{|c|}{ Total } \\
\hline & $\begin{array}{l}\text { Amostra } \\
\text { total }(n)\end{array}$ & $\begin{array}{c}\text { Baixa } \\
\text { estatura (n) }\end{array}$ & $\%$ & $\begin{array}{l}\text { Amostra } \\
\text { total }(n)\end{array}$ & $\begin{array}{c}\text { Baixa } \\
\text { estatura (n) }\end{array}$ & $\%$ & $\begin{array}{l}\text { Amostra } \\
\text { total }(n)\end{array}$ & $\begin{array}{c}\text { Baixa } \\
\text { estatura (n) }\end{array}$ & $\%$ \\
\hline 10 & 92 & 1 & 1,09 & 110 & 4 & 3,64 & 202 & 5 & 2,48 \\
\hline 11 & 920 & 25 & 2,72 & 749 & 11 & 1,47 & 1669 & 36 & 2,16 \\
\hline 12 & 1234 & 42 & 3,40 & 1109 & 37 & 3,34 & 2343 & 79 & 3,37 \\
\hline 13 & 1259 & 59 & 4,69 & 1132 & 33 & 2,92 & 2391 & 92 & 3,85 \\
\hline 14 & 1193 & 43 & 3,60 & 1063 & 42 & 3,95 & 2256 & 85 & 3,77 \\
\hline 15 & 919 & 33 & 3,59 & 922 & 37 & 4,01 & 1841 & 70 & 3,80 \\
\hline 16 & 647 & 19 & 2,94 & 698 & 36 & 5,16 & 1345 & 55 & 4,09 \\
\hline 17 & 464 & 20 & 4,31 & 407 & 29 & 7,13 & 871 & 49 & 5,63 \\
\hline 18 & 165 & 7 & 4,24 & 133 & 8 & 6,02 & 298 & 15 & 5,03 \\
\hline Total & 6893 & 249 & 3,61 & 6323 & 237 & 3,75 & 13216 & 486 & 3,68 \\
\hline
\end{tabular}



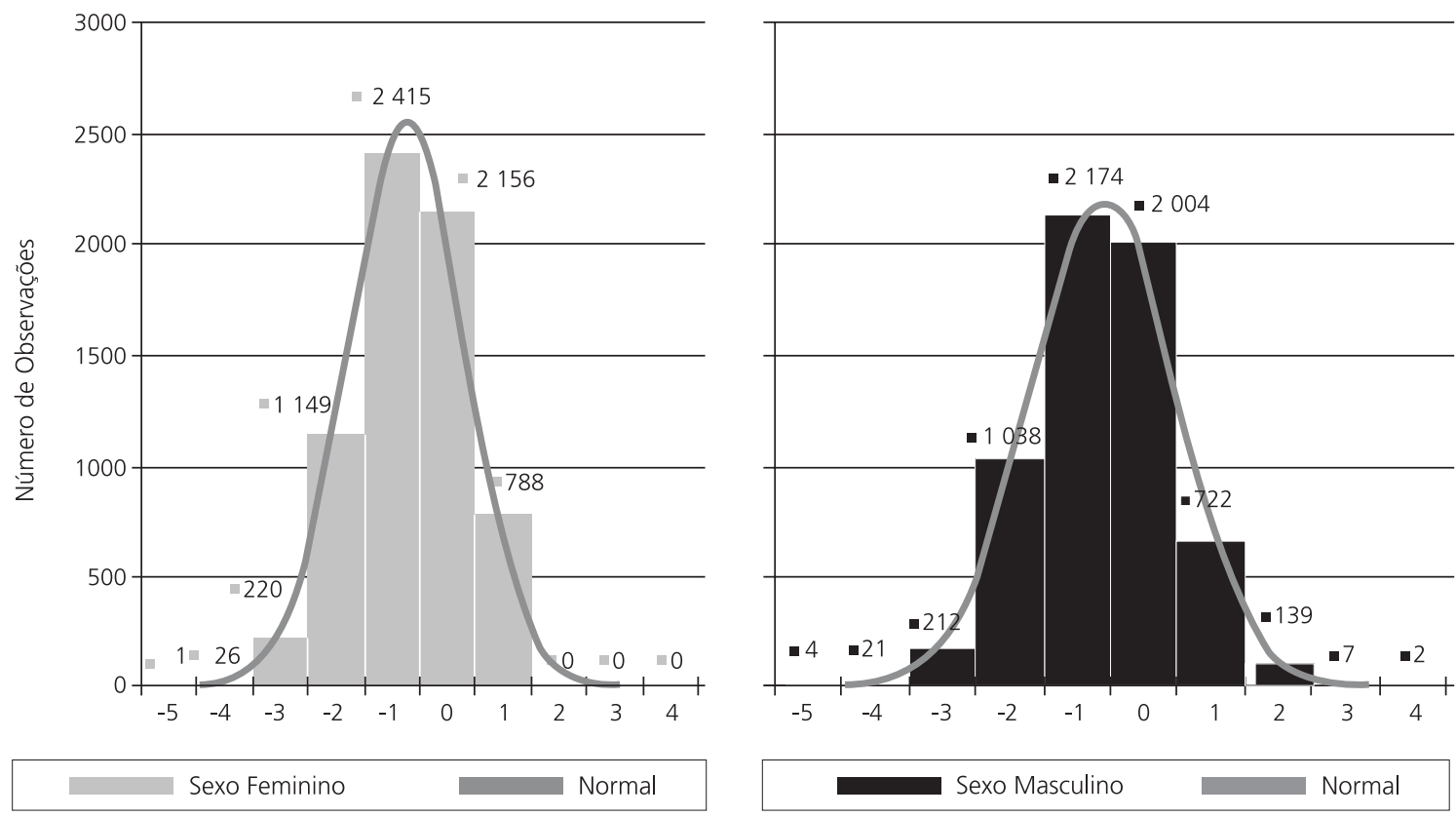

Distribuição do Índice Altura para a Idade (Escore-Z)

Figura 1. Distribuição do índice estatura para a idade de escolares da rede pública estadual no Paraná, em comparação com o padrão de normalidade, por sexo. Paraná, 2004

Na Figura 2 estão representadas as curvas de medianas de estatura e as dos pontos de corte inferior e superior (percentis 5 e 95) de escolares do sexo feminino e masculino deste estudo, da curva de referência $\mathrm{CDC}^{7}$, além das medianas da Pesquisa Nacional sobre Saúde e Nutrição - Região Sul ${ }^{6}$.

Nos escolares do sexo feminino no Paraná os valores do percentil 5 são semelhantes aos do CDC nas idades de 10,11, 12 e 18 anos, mantendo-se próximos aos mesmos nas demais idades, porém inferiores. Os valores do percentil 95 são superiores nos escolares do sexo feminino nas idades de 10 e 11 anos, havendo um encontro com a curva do CDC na estatura final (18 anos). Nos escolares do sexo masculino, os valores do percentil 5 são superiores ao CDC apenas aos 11 anos, e o percentil 95 é superior ao desta referência nas idades de 10, 11, 12, 13 e 14 anos, sendo inferiores nas demais e aproximando-se do CDC na idade de 18 anos $^{7}$.

Observa-se que a mediana da estatura de escolares no Paraná é maior, em relação à PNSN-Região Sul, em todas as idades e em ambos os sexos. Percebe-se também que as medianas nas faixas etárias de 10 e 11 anos são superiores aos valores do CDC (2000) ${ }^{7}$.

A Tabela 2 quantifica essas diferenças na estatura, pela comparação das medianas de escolares no Paraná com as medianas obtidas pela PNSN - Região Sul 15 anos antes deste estudo. Mostra também a intensidade do déficit de estatura pela comparação dos dados dos escolares deste estudo com as medianas de estatura da referência $\mathrm{CDC}^{7}$.

Em relação à PNSN, no intervalo entre 10 e 18 anos, a diferença entre as medianas de estatura foi positiva e variou, no sexo feminino de $2,70 \mathrm{~cm}$ a $6,30 \mathrm{~cm}$ e, no sexo masculino de $3,70 \mathrm{~cm}$ a $5,50 \mathrm{~cm}$. Alterações quanto à intensidade do crescimento foram observadas, porém as diferenças se mantiveram positivas para todas as idades em ambos os sexos. Aos 11 anos, no sexo feminino foi observada a maior diferença positiva $(6,30 \mathrm{~cm})$, enquanto no sexo masculino a maior diferença em relação à PNSN - Região Sul para mesma idade e sexo, foi aos 11 e 15 anos $(5,50 \mathrm{~cm})$. 


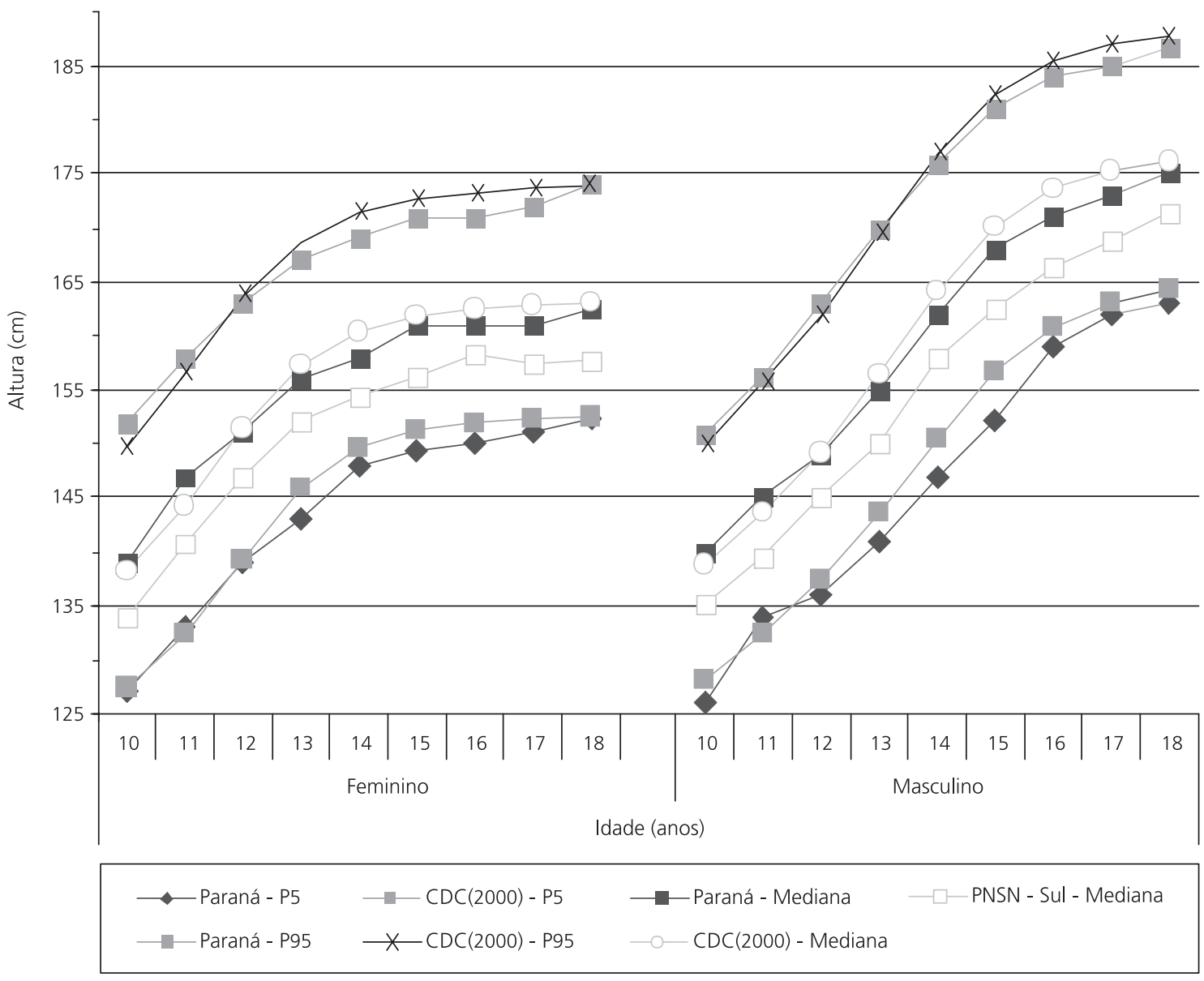

Figura 2. Medianas de crescimento e percentis 5 e 95 para escolares no Paraná e CDC (2000) e mediana de estatura para a PNSN Região Sul, por sexo.

As menores diferenças foram observadas aos 16 anos, no sexo feminino, mostrando-se $2,70 \mathrm{~cm}$ superior a PNSN e, aos 18 anos no sexo masculino, a diferença positiva foi de $3,70 \mathrm{~cm}$.

Em relação aos valores da referência $\mathrm{CDC}^{7}$ as medianas foram superiores nas idades de $10 \mathrm{e}$ 11 anos, na magnitude de $1,01 \mathrm{~cm}$ e $3,02 \mathrm{~cm}$, respectivamente, no sexo feminino, e no masculino em 1,38 e 1,48cm. Para as demais idades observou-se a presença de valores sempre mais baixos, variando de $-0,05 \mathrm{~cm}$ a $-2,29 \mathrm{~cm}$ no sexo masculino e $-0,19 \mathrm{~cm}$ a $-2,39 \mathrm{~cm}$ no sexo feminino. Para o sexo masculino a menor e a maior diferença negativa dos escolares do estudo em relação aos valores da referência CDC, ocorreram nas idades de 12 e 17 anos e foram de, respectivamente, $-0,05 \mathrm{~cm}$ e $-2,29 \mathrm{~cm}$. No sexo feminino, isso ocorreu aos 12 e 14 anos e foi, respectivamente, $-0,19 \mathrm{~cm}$ $\mathrm{e}-2,39 \mathrm{~cm}$ inferiores às medianas de crescimento do $C D C^{7}$.

Para verificar a relação entre a estatura dos escolares e o desenvolvimento social e econômico dos municípios avaliados aplicou-se o diagrama de dispersão da estatura versus IDH. O teste ilustrou a associação positiva entre estas variáveis, pois foi caracterizada no cálculo do coeficiente de correlação de Pearson $(r=0,37)$, sendo significativo sob o nível de $5 \%$. Com este resultado, ficou evidenciada a tendência de que em municípios mais desenvolvidos a estatura média dos adolescentes é maior. 
Tabela 2. Diferenças na estatura mediana de escolares da rede pública estadual no Paraná em relação à PNSN - região sul e em relação aos valores da referência CDC (2000), por sexo. Paraná, 2004.

\begin{tabular}{|c|c|c|c|c|c|c|}
\hline \multirow{2}{*}{$\begin{array}{r}\text { Sexo } \\
\text { Idade } \\
\text { (anos) }\end{array}$} & \multicolumn{6}{|c|}{ Feminino } \\
\hline & $\begin{array}{l}\text { Estatura mediana } \\
\text { Escolares no Paraná }\end{array}$ & $\begin{array}{l}\text { Estatura mediana } \\
\text { PNSN - Região Sul }\end{array}$ & $\begin{array}{l}\text { Diferenças na } \\
\text { estatura }(\mathrm{cm})\end{array}$ & $\begin{array}{l}\text { Estatura mediana } \\
\text { Escolares no Paraná }\end{array}$ & CDC (2000) & $\begin{array}{c}\text { Diferenças na estatura } \\
\text { Escolares no Paraná }\end{array}$ \\
\hline 10 & 139,00 & 133,90 & $+5,10$ & 139,00 & 137,99 & $+1,01$ \\
\hline 11 & 147,00 & 140,70 & $+6,30$ & 147,00 & 143,98 & $+3,02$ \\
\hline 12 & 151,00 & 146,90 & $+4,10$ & 151,00 & 151,19 & $-0,19$ \\
\hline 13 & 156,00 & 152,10 & $+3,90$ & 156,00 & 157,16 & $-1,16$ \\
\hline 14 & 158,00 & 154,30 & $+3,70$ & 158,00 & 160,39 & $-2,39$ \\
\hline 15 & 161,00 & 156,20 & $+4,80$ & 161,00 & 161,86 & $-0,86$ \\
\hline 16 & 161,00 & 158,30 & $+2,70$ & 161,00 & 162,55 & $-1,55$ \\
\hline 17 & 161,00 & 157,50 & $+3,50$ & 161,00 & 162,91 & $-1,91$ \\
\hline \multirow[t]{2}{*}{18} & 162,50 & 157,70 & $+4,80$ & 162,50 & 163,12 & $-0,62$ \\
\hline & \multicolumn{6}{|c|}{ Masculino } \\
\hline 10 & 140,00 & 135,20 & $+4,80$ & 140,00 & 138,62 & $+1,38$ \\
\hline 11 & 145,00 & 139,50 & $+5,50$ & 145,00 & 143,52 & $+1,48$ \\
\hline 12 & 149,00 & 145,00 & $+4,00$ & 149,00 & 149,05 & $-0,05$ \\
\hline 13 & 155,00 & 150,00 & $+5,00$ & 155,00 & 156,09 & $-1,09$ \\
\hline 14 & 162,00 & 157,90 & $+4,10$ & 162,00 & 163,84 & $-1,84$ \\
\hline 15 & 168,00 & 162,50 & $+5,50$ & 168,00 & 169,94 & $-1,94$ \\
\hline 16 & 171,25 & 166,50 & $+4,75$ & 171,25 & 173,51 & $-2,26$ \\
\hline 17 & 173,00 & 168,80 & $+4,20$ & 173,00 & 175,29 & $-2,29$ \\
\hline 18 & 175,00 & 171,30 & $+3,70$ & 175,00 & 176,16 & $-1,16$ \\
\hline
\end{tabular}

NOTA: PNSN - Pesquisa Nacional sobre Saúde e Nutrição6; CDC (2000) - Centers for Disease Control and Prevention7; Diferenças na estatura mediana equivalem ao valor da mediana de estatura dos escolares da rede pública no Paraná, menos o valor da mediana da PNSN - Região Sul ou da mediana dos valores da referência (CDC/2000) para mesma idade e sexo. O símbolo (+) representa o ganho na estatura. $O$ símbolo (-) representa o déficit de estatura.

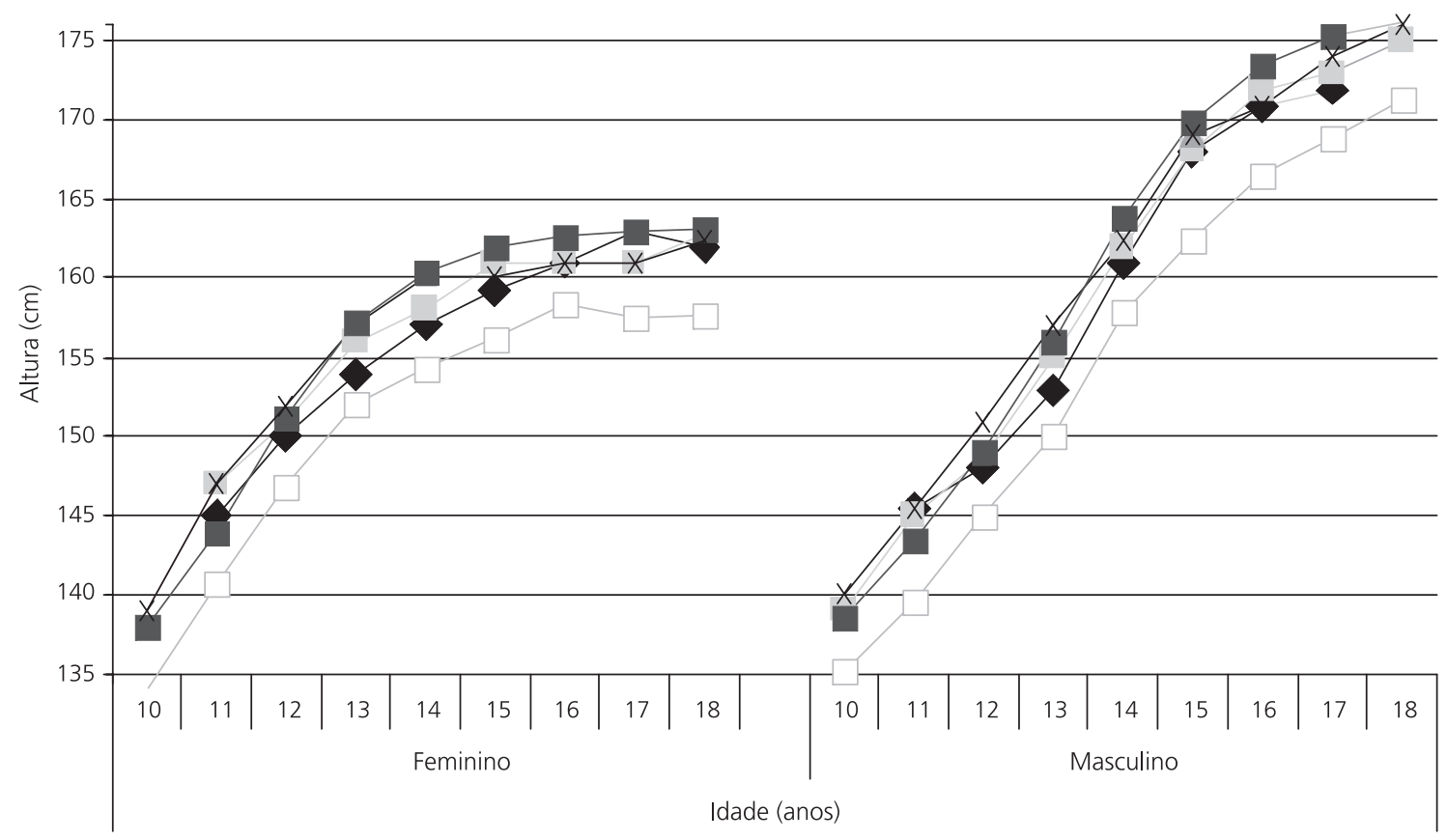

$\longrightarrow$ IDH 6,5 $\longrightarrow$ IDH 7,5 $\longrightarrow$ IDH 8,5 $\longrightarrow$ CDC (2000) $\square$ PNSN, Região Sul (1990)

Figura 3. Medianas de estatura de escolares da rede pública do Paraná, segundo extremos de desenvolvimento humano (IDH) municipal e em comparação com o CDC (2000) e a PNSN - Região Sul. Paraná, 2004. 
A Figura 3 apresenta as medianas de estatura estratificadas segundo grupos de municípios com os menores e maiores IDH e compara estes resultados com a referência $\mathrm{CDC}^{7}$ e a PNSN (1989) - Região Sul. Para esta análise, os municípios foram agrupados no valor central de IDH, por exemplo, o estrato de IDH de 6,5 engloba os municípios com IDH maiores ou iguais a 6,25 e menores que 6,75 . Os demais grupos de $\operatorname{IDH}(7,0$; 7,5 e 8,0) distribuíram-se entre IDHs de 6,5 e 8,5. Pela análise gráfica é possível concluir que as medianas de estatura dos escolares do Paraná são superiores às da PNSN Região Sul, para todos os índices de desenvolvimento humano.

Quando se comparam com as medianas do $C D C$, verifica-se que os escolares de municípios com IDH 6,5 apresentam estaturas a partir dos 12 anos, sempre mais baixas do que a referência, especialmente no sexo feminino. Já naqueles municípios com IDH 8,5 as medianas de estatura estão muito próximas e, em algumas idades (10 a 13 anos), até mais altas do que as do CDC? .

\section{I S C U S S Ã O}

A distribuição do índice estatura para a idade, em escores-Z, mostrou que para os escolares de 10 a 18 anos deste estudo, a incidência de baixa estatura é muito próxima do esperado, sendo apenas 1,38\% superior aos $2 Z$ da referência $\mathrm{CDC}^{7}$. Esta dispersão é considerada baixa.

A partir do teste de Kolmogorov-Smirnov, concluiu-se que a distribuição do índice estatura para a idade foi normal para o total de escolares, para cada faixa etária e sexo. Esse fato demonstra que, embora tenham sido encontradas dispersões nas taxas de baixa e alta estatura em algumas faixas etárias para ambos os sexos, a distribuição foi normal, ou seja, os resultados não excluem a hipótese de que os valores obtidos têm distribuições normais semelhantes aos da população referência.

Em relação à PNSN-Região Sul, observa-se uma diferença positiva de $4,80 \mathrm{~cm}$ na estatura para o sexo feminino na idade de 18 anos e de $3,70 \mathrm{~cm}$ no sexo masculino para a mesma idade.
Enquanto o déficit de estatura da PNSN-Região Sul em relação à referência $C D C^{7}$, na estatura final, foi em média de $5,42 \mathrm{~cm}$ e $4,86 \mathrm{~cm}$ para o sexo feminino e masculino, respectivamente, entre os escolares deste estudo, o déficit foi de apenas $0,62 \mathrm{~cm}$ no sexo feminino e de $1,16 \mathrm{~cm}$ no sexo masculino.

Embora a definição da amostra do presente estudo não permita inferências para o total de escolares da rede pública do Paraná, o número de indivíduos estudados, residentes em todas as regiões do Estado, permite supor que é possível encontrar resultados semelhantes no restante dessa população.

Pelos mesmos motivos e, mesmo levando-se em consideração que as comparações das estaturas medianas dos escolares foram efetuadas com estudo na Região Sul (PNSN) e não especificamente com dados anteriores do Estado, pode-se supor que as diferenças positivas encontradas sejam conseqüências de melhorias socioeconômicas ocorridas no Paraná nas últimas décadas. De acordo com dados da Pesquisa Nacional por Amostra de Domicílios (PNAD), diminuíram as taxas de analfabetismo infantil e adulto, aumentou a escolaridade média em anos de estudo para pessoas com idade superior a 25 anos e a taxa de mortalidade infantil reduziu de 33,50\% em 1990, para 16,47 por mil nascidos-vivos em $2004^{10}$. Em relação às condições de moradia ocorreram várias melhorias neste período, tais como: o acesso adequado ao escoamento de esgoto sanitário, à água canalizada, à coleta de lixo adequada, à energia elétrica, a bens de consumo duráveis como telefone fixo, geladeira, filtro de água e fogão ${ }^{11}$.

Melhorias no Estado são observadas também ao serem avaliados os Índices de Desenvolvimento Humano em que houve um aumento em todas as categorias (longevidade, educação e renda), modificando o índice geral de 0,711 para 0,787 no período 1991 a 2000². A constatação de que os escolares de municípios com IDH 8,5 apresentaram as medianas de estatura muito próximas e, até mais altas em algumas idades do 
que a referência CDC, leva a supor que os adolescentes com acesso às melhorias citadas podem alcançar a referência para crescimento.

Portanto, mesmo levando-se em consideração tratar-se de uma pesquisa com amostra não probabilística, embora relevante do ponto de vista do número de indivíduos investigados, os resultados encontrados mostram uma diferença positiva nas medianas de estatura dos escolares em relação aos dados encontrados pela PNSN (1989), para as mesmas faixas etárias e sexo. $E$, mesmo não dispondo de outros estudos específicos e representativos dessa população no Paraná, os dados sobre melhorias nas condições socioeconômicas da população nas últimas décadas, sugerem uma possível contribuição para as medianas de estatura nos escolares da amostra.

Com isso, não se descartam, contudo, as diferenças regionais. Se nas últimas décadas, o Estado teve um desenvolvimento que contribuiu para a modernização de sua base produtiva, muitos municípios encontram-se excluídos tanto econômica como socialmente desses benefícios ${ }^{13}$. Essa situação é evidenciada nos resultados das medianas de estatura dos escolares de municípios com IDH 6,5, cujos valores são sempre mais baixos do que a referência.

Ressalta-se, assim, a necessidade e a importância de estudos representativos, tanto transversais como longitudinais, que estabeleçam a tendência e o perfil de crescimento dessa população. Por outro lado, no planejamento de políticas públicas, é importante que as diferenças regionais sejam consideradas apontando demandas específicas no seu processo de implantação e implementação. Essa observação é relevante, sobretudo quando se considera o conceito atual de eqüidade, que aponta para a necessidade de um tratamento diferenciado de situações, visando à superação de desigualdades econômicas, sociais, de gênero e étnicas, que ao longo da história de um território se materializam interferindo no processo saúde-doença e na determinação do estado nutricional de sua população.

\section{A GRADECIMENTOS}

Ao Centro Colaborador em Alimentação e Nutrição-Região Sul, pelo apoio financeiro ao projeto; à Profa. Sílvia do Amaral Rigon, do Departamento de Nutrição da Universidade Federal do Paraná, pela participação nas discussões que resultaram em importantes contribuições na construção deste artigo.

\section{COLABORADORES}

S.T.S.P. AMORIM coordenou o projeto "Estado nutricional de escolares no Paraná" do qual este estudo é parte, responsável pela análise e interpretação dos dados deste estudo, pela sistematização, interpretação e pela publicação dos dados de todo o projeto. A.G.M. RODRIGUES responsável pela sistematização contribuiu com a análise e a interpretação dos dados. M.C. STOLARSKI responsável pela concepção do estudo e pela coleta dos dados.

\section{REFER Ê N CIAS}

1. Varela-Silva MI, Bogin B. Growth as a measure of socioeconomic inequalities and poor living conditions among Portuguese, Cape Verdean-Portuguese, and Cape Verdean children, between 1993 and 2001. Proceedings of the $1^{\text {st }}$ Lusophone Africa Conference: Intersections Between the Social Sciences, 2003, Cornel University - NY. [cited 2006 Jun]. Available from: <http://www.kyle.aem.cornell. edu/lusopaps/Varela-Silva_\&_Bogin.pdf>.

2. Ulijaszek SJ. Between-population variation in pre-adolescent growth. In: Waterlow JC, Schürch B. Causes and mechanisms of linear growth retardation. London; 1993.

3. Freedman DS, Khan LK, Serdula MK, Srinivasan SR, Berenson GS. Secular trends in height among children during 2 decades: the Bogalusa Heart Study. Arch Pediatr Adolesc Med. 2000; 154(Pt 2): 155-61.

4. Monteiro CA, Benicio MHD'A, lunes R, Gouveia NC, Taddei JAAC, Cardoso MAA. ENDEF e PNSN: para onde caminha o crescimento físico da criança brasileira? Cad Saúde Pública. 1993; 9(Suppl1): 85-95.

5. Monteiro CA, Benício MHD, Gouveia NC. Evolução da altura dos brasileiros. In: Monteiro CA. Velhos e novos males da saúde no Brasil: a evolução do 
país e de suas doenças. 2a. ed. São Paulo: Hucitec; 2000. p. 126-40.

6. Brasil. Ministério da Saúde. Instituto de Alimentação e Nutrição. Pesquisa Nacional sobre Saúde e Nutrição. Perfil de crescimento da população brasileira de 0 a 25 anos. Brasília; 1990.

7. National Center for Health Statistics. 2000 CDC growth charts for the United States: methods and development. Washington; 2000. p.201. Series Report 11, 246.

8. Dean AG, Dean JA, Burton AH, Dicker RC. Epi Info, version 6bc: a word processing, database, and statistics program for epidemiology on microcomputers. Georgia: Center for Disease Control and Prevention; 1997.

9. Morcillo AM. Teste de Kolmogorov-Smirnov. Campinas: Unicamp. [acesso 2006 nov 15]. Disponível em: <http://www.fcm.unicamp.br/ centros/ciped/mp639/Teste \%20de\%20 Kolmogorov.pdf>.

10. Paraná. Secretaria de Estado do Planejamento e Coordenação Geral. Instituto Paranaense de Desenvolvimento Econômico e Social. Coeficientes de mortalidade infantil, materna e geral Paraná. [acesso 2006 nov 05]. Disponível em: <http:// www.ipardes.gov.br/pdf/indices/coeficiente_ mortalidade.pdf>.

11. Paraná. Secretaria de Estado do Planejamento e Coordenação Geral. Instituto Paranaense de Desenvolvimento Econômico e Social. Índice de desenvolvimento humano municipal (IDH-M) Paraná. [acesso 2006 nov 15]. Disponível em: <http://www.ipardes.gov.br/>.

12. Instituto de Estudos do Trabalho e Sociedade. Dados e tabulações da série histórica da Pesquisa Nacional por Amostra de Domicílios, 1992-2004. [acesso 2006 nov 5]. Disponível em: <http://www. iets.org.br/>.

13. Instituto Paranaense de Desenvolvimento Econômico e Social. Paraná: diagnóstico social e econômico. Curitiba; 2003.

Recebido em: 22/6/2007

Versão final reapresentada em: 7/8/2008 Aprovado em: 12/11/2008 